\title{
La arquitectura escolar de José María García de Paredes en Granada. Un prototipo, tres escuelas
}

\section{School architecture of José María García de Paredes in Granada. One prototype, three schools}

S. Castillo Hispán $^{(*)}$, E. Valero Ramos ${ }^{(* *)}$

RESUMEN

José María García de Paredes, construyó entre 1964 y 1967 tres escuelas en Granada, una arquitectura comprometida, que sigue sorprendiendo cincuenta años después por la vigencia y rotundidad de los principios que las originaron.

Estos son los únicos edificios escolares que García de Paredes construyó en su carrera, en ellos sintetizo un discurso completo sobre los nuevos espacios docentes que se demandaban por la sociedad de la época. Y lo hizo en unas circunstancias especialmente complicadas en las que el proyecto surge como solución a un problema casi imposible de tiempos, economía y construcción. Aunque nunca fueron pensadas para estar en servicio tanto tiempo y construidas con materiales humildes, han cumplido de una manera ejemplar los cincuenta años, y lo hacen en pleno funcionamiento, sin haber sufrido importantes modificaciones en las formas y principios que las generaron.

Palabras clave: García de Paredes; arquitectura docente; escuelas en Granada; arquitectura racionalista.

\section{ABSTRACT}

José María García de Paredes built three schools in Granada between 1964 and 1967. It was an architecture socially committed that carries on surprising fifty years later due to the clarity of its principles.

These are the only school buildings that García de Paredes built during his career. In these buildings he presented a comprehensive approach about the educational architecture that was demanded in the society at the time. He was able to carry out these projects despite the hard circumstances in terms of construction, economy and time. These buildings were not intended to keep working during a long time and were made with simple materials. Yet, they have been successfully performing during fifty years without having changed its principles and shape.

Keywords: García de Paredes; educational architecture; schools in Granada; rationalism; rationalist architecture.

(*) Universidad de Málaga (España).

(**) Universidad de Granada (España).

Persona de contacto/Corresponding author: sergioch@uma.es (S. Castillo Hispán)

Cómo citar este artículo/Citation: Castillo Hispán, S., Valero Ramos, E. (2016). La arquitectura escolar de José María García de Paredes en Granada. Un prototipo, tres escuelas. Informes de la Construcción, 68(541): e138, doi: http://dx.doi.org/10.3989/ic.15.133.

Licencia / License: Salvo indicación contraria, todos los contenidos de la edición electrónica de Informes de la Construcción se distribuyen bajo una licencia de uso y distribución Creative Commons Reconocimiento no Comercial 3.o. España (cc-by-nc). 


\section{INTRODUCCION}

José María García de Paredes es sin lugar a dudas uno de los arquitectos más destacados de la segunda generación de postguerra (1). Un grupo de jóvenes formados en la escuela de arquitectura de Madrid, que continuarán la labor iniciada por Oiza, de la Sota, Fisac, Cabrero y Aburto.

Nace en Sevilla en el año 1924 e inicia la carrera en 1944, por recomendación de Casto Fernández Shaw. Titulándose en 1950, como miembro destacado de la 100 promoción, junto con su compañero y amigo Rafael de la Hoz.

Completa su formación con numerosos viajes de estudios por Inglaterra, Irlanda, Francia, Holanda, Italia, Suecia, Noruega, Dinamarca y Alemania.

En 1956 se traslada a Roma como pensionado de la Real Academia de Bellas Artes de San Fernando hasta 1958, beca que gana por su proyecto de pabellón español en la Bienal de Venecia. Esta oportunidad le permitirá establecer importantes lazos con numerosas personalidades del panorama arquitectónico europeo, como Gio Ponti en el que encontrará un interés constante por su obra.

En esta estancia afianza su amistad con Javier Carvajal, con quien construye en 1957 el Panteón de los Españoles en la capital italiana y el pabellón de España en la en la XI Trienal de Milán, con el que obtienen la Medalla de Oro del evento.

Este periodo de formación fuera de España le permite establecer una apropiada distancia con el cerrado panorama arquitectónico nacional y encontrar nuevas referencias.

En este sentido cabe destacar, por la influencia que tendrá en su obra posterior, el extenso viaje que realizo por el norte de Europa en su segundo año de pensionado. Un viaje que le permitió conocer de primera mano las importantes aportaciones realizadas en todos los campos de la arquitectura por los maestros nórdicos.

A principios de la década de los 60 García de Paredes es un arquitecto que ya ha realizado numerosos proyectos de relevancia a nivel nacional. Un arquitecto, que apuesta por una arquitectura decididamente comprometida con los principios de la modernidad, pero a su vez atenta a las circunstancias concretas de cada encargo, para el que cada proyecto constituye una nueva oportunidad de investigación y desarrollo.

Sus primeros encargos se entremezclan con los últimos años de formación, y los comparte con Rafael de la Hoz.

Su primera obra es la Cámara de Comercio e Industria de Córdoba, construida entre 1950 y 1954, en la que cuentan con la colaboración del escultor Jorge Oteiza en una intención clara de incorporar el arte moderno dentro del proyecto de arquitectura (2).

Tras la Cámara de Comercio, y antes de su pensionado en Roma, realiza el encargo para el Colegio Mayor Aquinas, por el que es Premio Nacional de arquitectura en 1956, que proyecta también con Rafael de la Hoz. Esta obra parece adelantar muchas de las características de la línea de trabajo que seguirá en adelante, una delicada puesta en el lugar, y un delicado trabajo con los materiales y acabados.
En 1964, año en el que proyecta las escuelas en Granada, acaba de terminar el poblado Dirigido de Almendrales con Corrales, Molezún y Carvajal, el último de esta serie de poblados en Madrid, en la misma línea de compromiso con la modernidad, pero cargado de referencias nórdicas en sus espacios y en el uso de los materiales.

Asimismo en el campo de la arquitectura docente estaba construyendo en Teruel la Escuela de Artes aplicadas (19631966) y acababa de terminar en Madrid la Escuela de Ingenieros de Telecomunicaciones (1960-1964), en colaboración con Javier Carvajal.
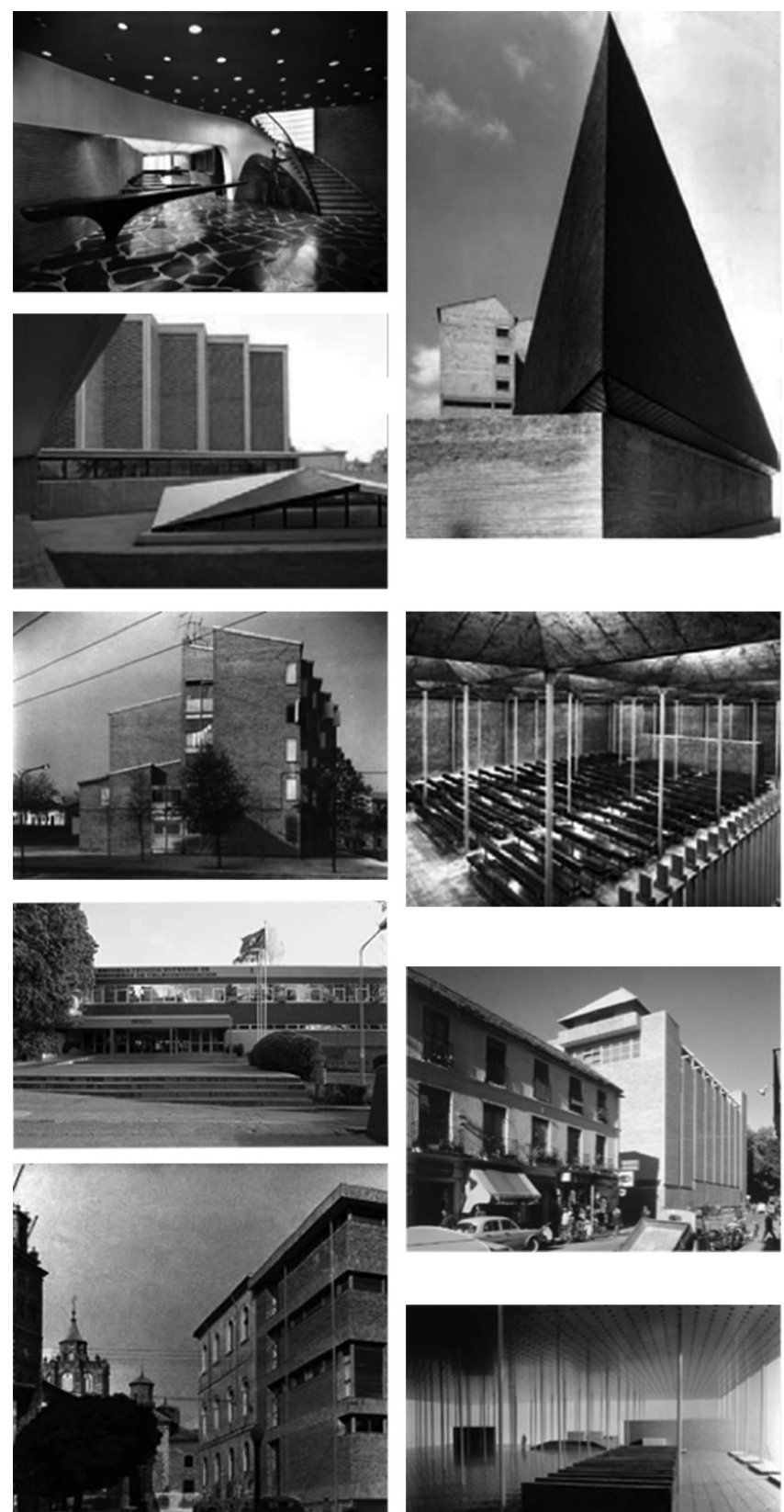

Figura 1. 1951-1952 Cámara de Comercio de Córdoba (2). 1953-1957 Colegio Mayor Aquinas en la Ciudad Universitaria, Madrid (1). 1958-1964 Poblado Dirigido de Almendrales, Madrid (3). 1960-1964 Escuela de Ingenieros de Telecomunicación, Madrid. 1963-1967 Escuela de Artes Aplicadas y Oficios Artísticos, Teruel -Fotografía de JMGP (4) - 1956-1960 Iglesia de Nuestra Señora de los Ángeles en Vitoria (5). 1961-1964 Centro parroquial de Almendrales (5). 1961-1964 Iglesia y Convento de Santa María de Belén en Málaga - Fotografía de A. Schommer (5)-. 1960 Maqueta para el concurso de San Esteban Protomártir en Cuenca (5). 
En estos proyectos, construidos entre 1958 y 1964 (Figura 1), se hace notable como García de Paredes ha construido un lenguaje personal e independiente, que se nutre de un conocimiento de primera mano de lo que está ocurriendo en Europa en ese momento, y de un exhaustivo método de trabajo e investigación, haciendo de cada proyecto un motivo nuevo de conocimiento que no se remite a los anteriores.

\section{LA PRIMERA ESCUELA EN EL ZAIDIN DE GRANADA}

García de Paredes proyectará y construirá su primera escuela en el barrio del Zaidín de Granada en 1964.

Con esta obra regresa a Granada tras la Exposición sobre Manuel de Falla que realizo en el año 1962 en el Monasterio de San Jerónimo con motivo el XI Festival Internacional de Música y Danza (6). En esta exposición utiliza de forma magistral la modulación, la austeridad y la prefabricación, claves proyectuales que empleará en las escuelas (Figura 2).

El encargo parte de la institución benéfico-docente Juan XXIII, con la condición expresa de que el edificio debería estar acabado en septiembre de ese mismo año, para albergar el siguiente curso académico a 1000 alumnos, de una de las zonas más deprimidas de la ciudad.
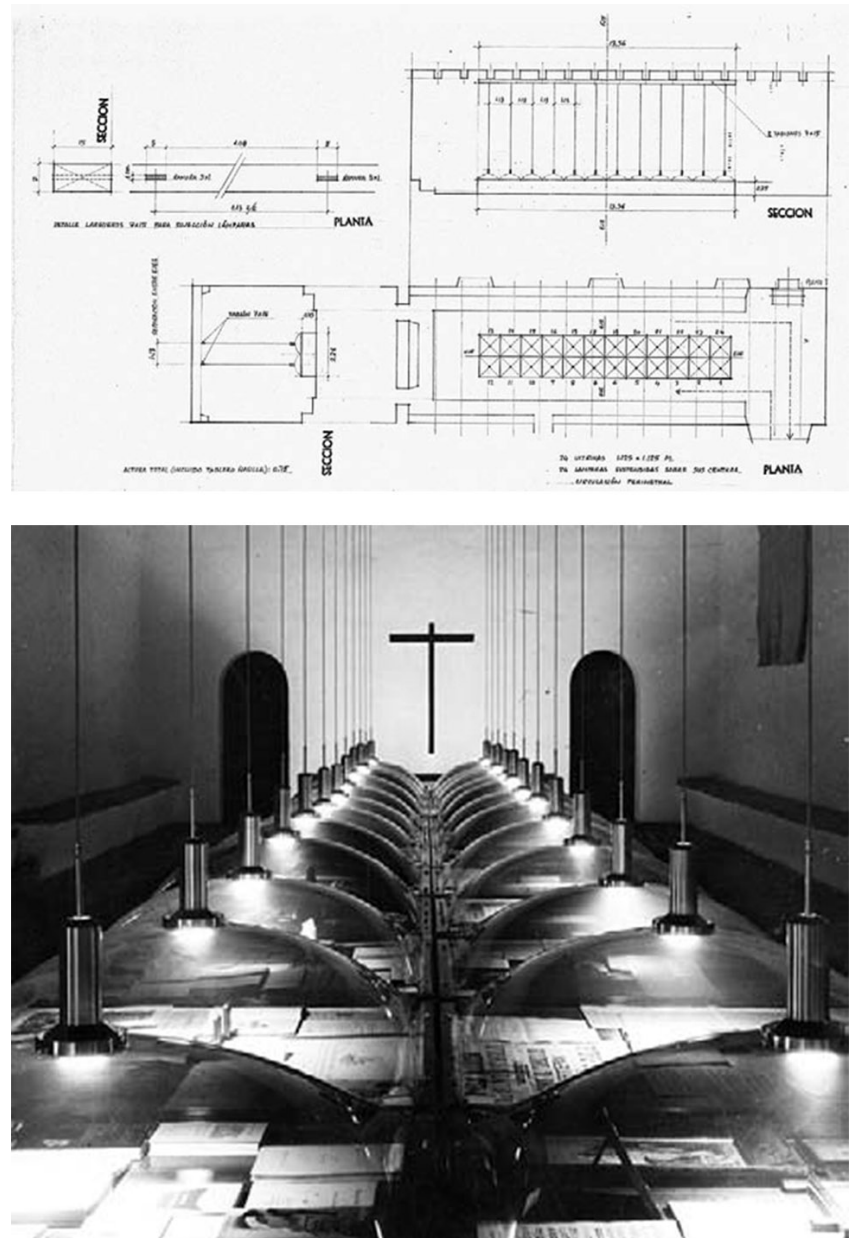

Figura 2. 1962 Exposición sobre Manuel de Falla en el Monasterio de San Jerónimo con motivo el XI Festival Internacional de Música y Danza -Fotografía de JMGP (4)-.
La solución propuesta para este primer colegio fue un edifico modular, capaz de dar respuesta a un grave problema de escolarización, con una enorme economía de medios, y en unos plazos ciertamente cortos. La solución propuesta fue considerada por la institución como prototipo para otras escuelas. Así repetirá el esquema con variaciones según el emplazamiento, en 1965 en los barrios de la Chana y el Albaicín.

Estas escuelas suponen sus primeros edificios construidos en Granada, la continuación lógica de la relación tan especial que estableció con esta ciudad, y que se había iniciado 1952 cuando conoce a Isabel de Falla.

Esta relación con Granada se prolongará de forma continuada a lo largo de toda su carrera. Desarrollará una obra no muy extensa, construyendo apenas sesenta edificios, trece de los cuales, y varios proyectos no construidos, están aquí.

Este primer encargo en el barrio del Zaidín de Granada es su primer proyecto de arquitectura escolar construido. Si bien ha trabajado previamente con espacios docentes en Escuela de Artes Aplicadas de Teruel y en la Escuela de Ingenieros de Telecomunicaciones, las cuestiones principales a resolver en este proyecto son totalmente diferentes.

Se propone un programa escolar con una apuesta por un modelo educativo nuevo y moderno, que debía ejecutarse con unos plazos muy ajustados y con una enorme economía de medios. García de Paredes resuelve el proyecto como un ejercicio de habilidad y oficio.

El problema principal que se establece como punto de partida en el proyecto, no es tanto el dar una respuesta urbana (Figura 3), sino dar solución al grave problema de escolarización existente, en poco tiempo y con escasos medios.

Los plazos que se cumplieron, tanto para el proyecto como para la construcción de las obras, fueron extraordinariamente cortos, el 13 de abril de 1964 se presentó el proyecto, el día 15 de mayo de inician las obras y el 2 de septiembre de ese
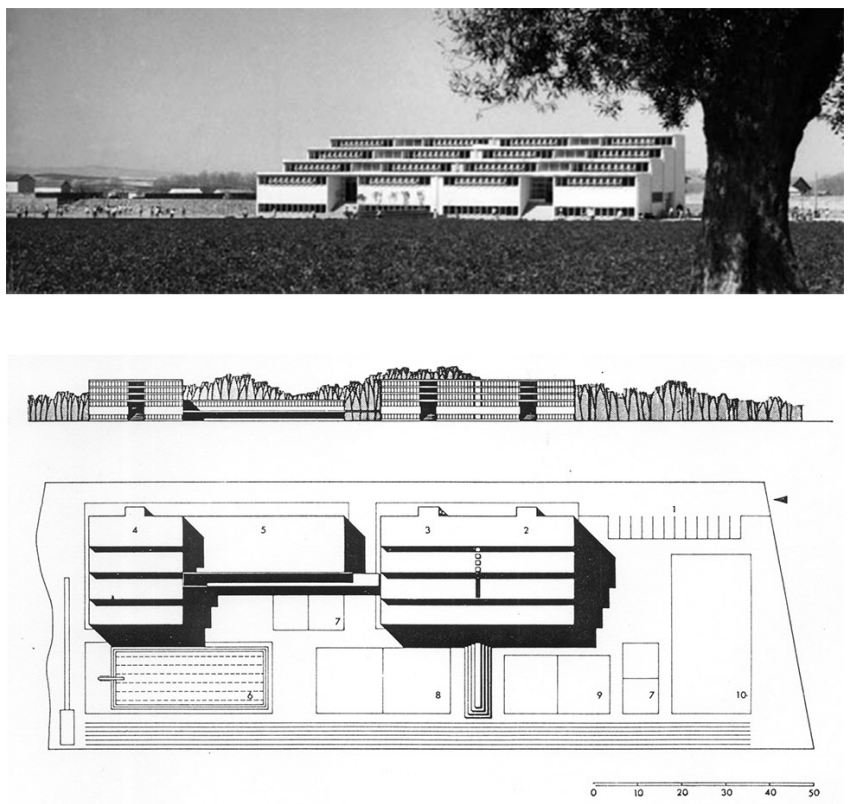

Figura 3. Centro de Enseñanza Media Juan XXIII en el Zaidín, plano de emplazamiento, 1964 -Fotografía de JMGP (4)-. 
año se inaugura el edificio. Como recoge en el Diario Ideal de Granada de 2 de septiembre de 1964:

«En el Zaidín ha surgido un hermoso edifico, en situación inmejorable, cara a la Sierra, con orientación hacia el mediodía, que por tratarse de un centro docente, del Patronato de la institución Juan XXIII, estará rodeado su campo con setos, arbolado y jardines que embellecerán todo el conjunto» (7).
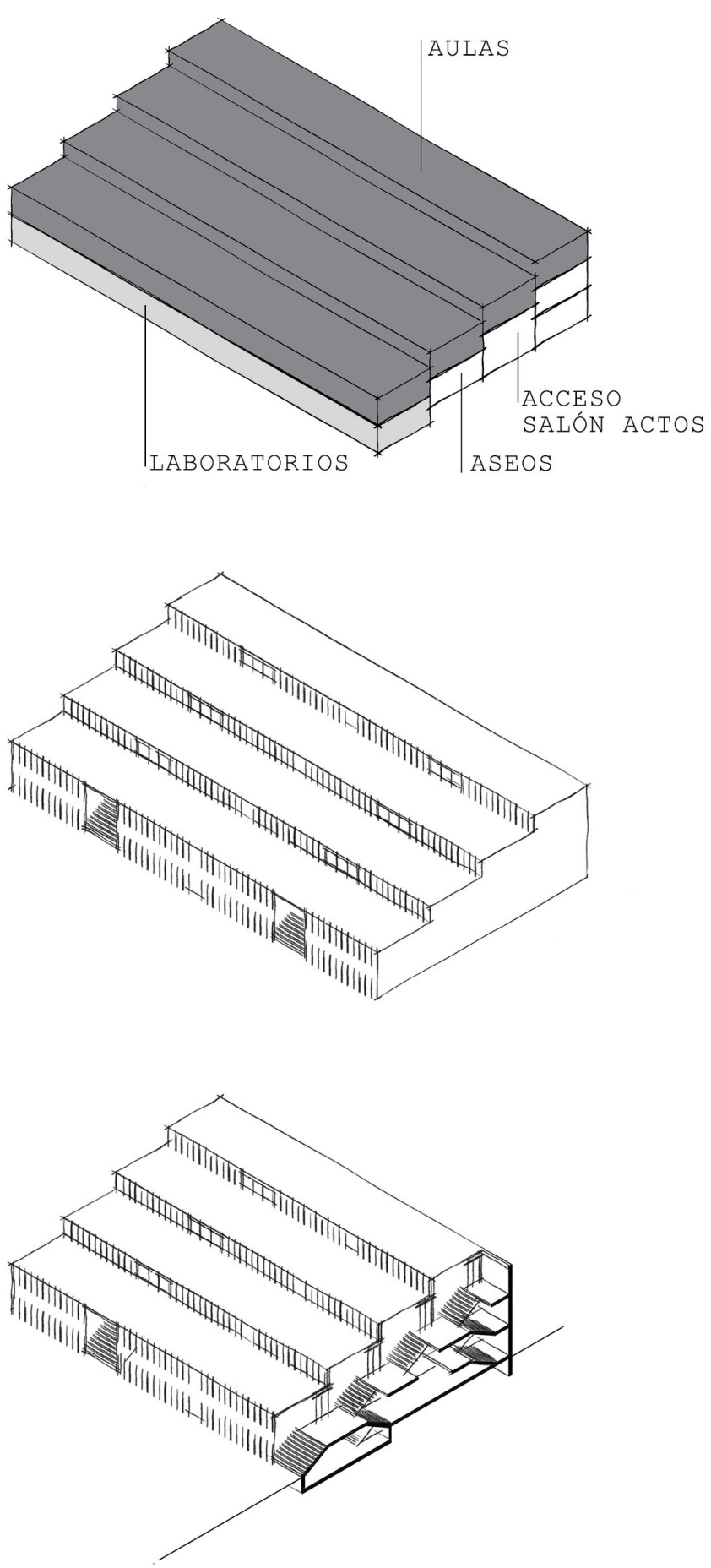

Figura 4. Centro de Enseñanza Media Juan XXIII, esquema de uso y funcionamiento. (Realizado por el autor).
La solución planteo una arquitectura clara, conceptual y con una enorme austeridad constructiva, que permitió realizar la obra dentro del plazo marcado. Una arquitectura perfectamente modulada y medida que resuelve el problema planteado con la mayor eficiencia posible (Figura 4).

Es así una solución extrapolable, un prototipo, que mediante una sección escalonada permita reducir al máximo la superficie de cerramiento y las circulaciones priorizando la correcta iluminación de todas las aulas (Figuras 5 y 6), como explica en el comienzo de la memoria:

«Suele haber, en casi todos los proyectos, un factor determinante de la solución arquitectónica que se adopta. Un factor que, a veces, termina por adquirir un papel tiránico en la ordenación de sacrificios de Ruskin. En el caso del Centro de Enseñanza Media Juan XXIII, este factor ha sido la rapidez de concepción y realización: en efecto, en el mes de mayo del 64 se contaba con un terreno de una hectárea, afortunadamente horizontal, y una masa de mil niños que tenían que entrar a clase en Octubre» (8).

\section{LA ESCUELA COMO PROTOTIPO}

Este esquema será repetido en 1965 en otras dos escuelas, confiriéndole al sistema ideado la condición de prototipo con la que había sido proyectado.

Una de ellas, promovida por la misma institución benéficodocente Juan XXIII, se construirá en el barrio de la Chana con pequeñas variaciones, derivadas de lo aprendido en la primera experiencia. La otra se levantará, por encargo de una Comunidad Religiosa Hijas de Cristo Rey en el Albaicín.

Estas escuelas construidas desde instituciones privadas en la década de los sesenta en Granada constituyen un trabajo ejemplar de escolarización de los sectores más deprimidos de la ciudad.

Estas instituciones benéfico-docentes apostaron por un modelo educativo nuevo y moderno, a la vanguardia de las corrientes de cambio existentes en ese momento en Europa. Como queda de manifiesto en la entrevista hecha al sacerdote reverendo Rogelio Macías, publicada a la presentación del proyecto en la Hoja del Lunes de Granada del 13 de abril de
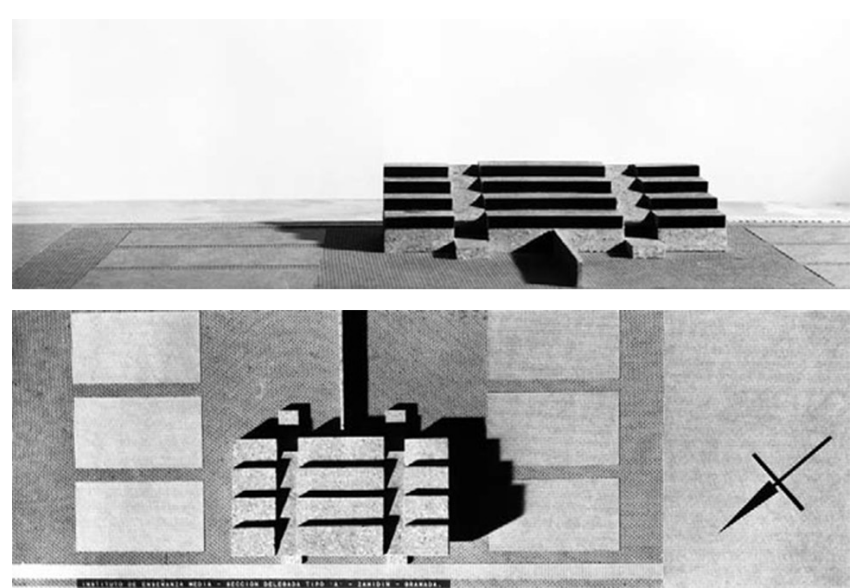

Figura 5. Centro de Enseñanza Media Juan XXIII, fotos de la maqueta (4). 

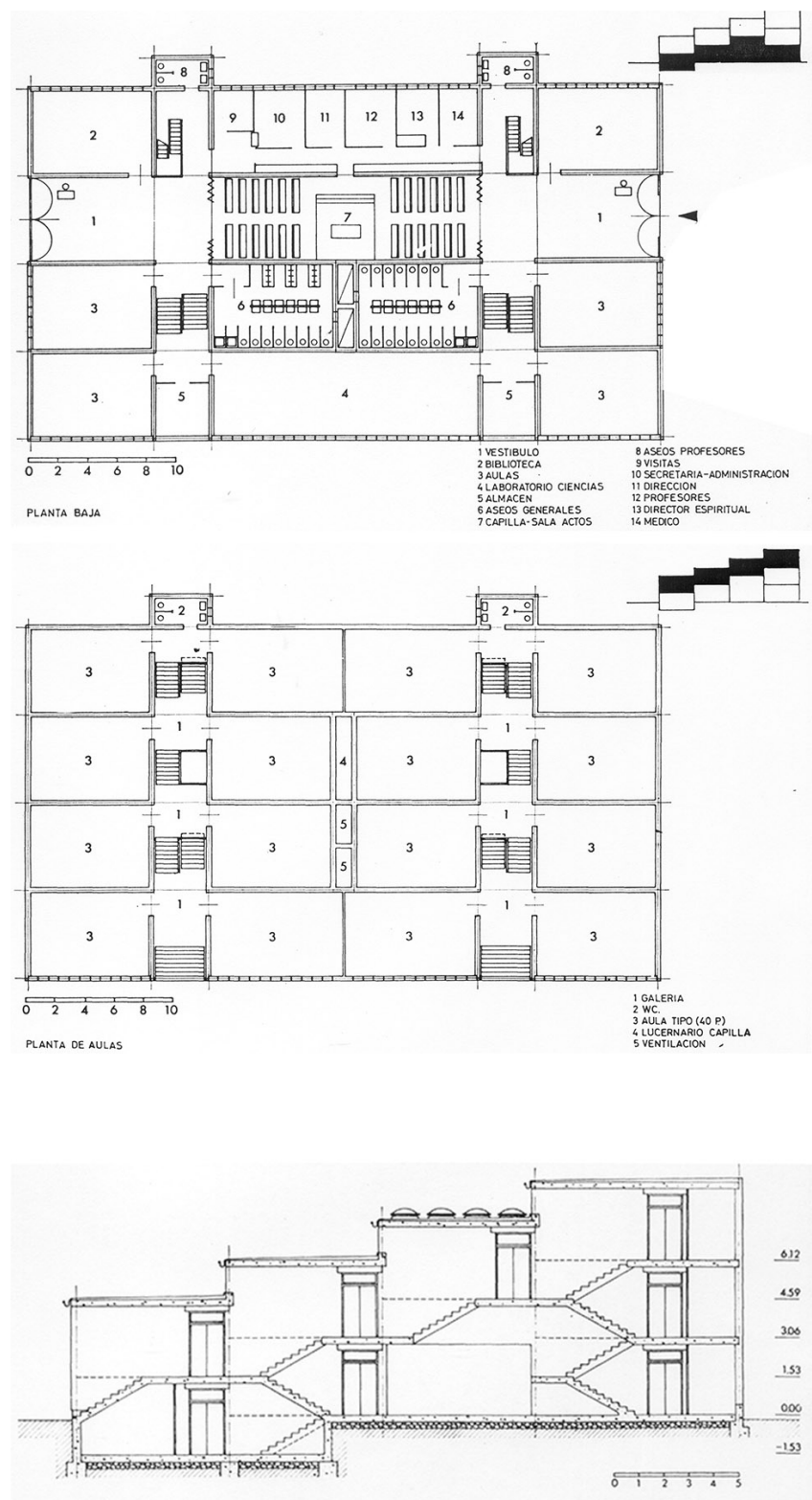

Figura 6. Centro de Enseñanza Media Juan XXIII, Planta baja, planta de aulas, sección por la galería de escaleras y esquema de uso y funcionamiento. (Base de datos del Instituto Andaluz de Patrimonio Histórico).

1964 , en la que no habla de arquitectura, sino de la función social del mismo y del moderno sistema educativo propuesto:

«No es un colegio para pobres, en el sentido peyorativo que se suele dar a esta frase, sino un centro modelo por su profesorado, instalación y orientación y al alcance de todas las familias trabajadoras... Quisiera invitar a todas las familias del Zaidín y pueblos cercanos a inscribir a sus hijos en este centro, dada la grave responsabilidad en la educación y enseñanza que tienen sobre ellos. Le ruego diga además que deseamos que se conozca la realidad de este nuevo centro, porque en la selección de alumnos serán los mejores los que ocupen los primeros puestos, y por mejores no son los que tengan más medios económicos, sino los más buenos e inteligentes, entre éstos preferimos a los que pertenezcan a familias trabajadoras y modestas, que no pueden costear un colegio» (7).

El compromiso con esta propuesta docente, y con la importantísima labor social que encierra, es total por parte de García de Paredes. El proyecto asume como labor principal el hacerlo posible, realizando una propuesta arquitectónica que sintetiza y articula los valores en nuevos espacios para la educación.

García de Paredes demuestra estar al día de las necesidades pedagógicas exigidas a las nuevas escuelas, aplicadas al proyecto, para convertir el aula en el espacio que concentra el germen necesario para el cambio.

El aula se convierte en el lugar más importante, un espacio limpio, flexible y perfectamente iluminado. Dotándola de las mejores condiciones para transmitir conocimiento, un entorno amable para el desarrollo de la infancia.

\section{LA REFERENCIA EUROPEA. LA ESCUELA MUNKEGARDS DE ARNE JACOBSEN}

Como expone La arquitectura de José M. García de Paredes. Ideario de una obra Las escuelas de García de Paredes guardan una clara conexión con la Escuela Munkegards de Jacobsen (9).

Las escuelas de Jacobsen se convirtieron en un referente dentro de las nuevas edificaciones docentes que se realizaron en la década de los 60.

García de Paredes las visitó en 1957 en el extenso viaje que realizó por Europa el último año de su pensionado en Roma (Figura 7).

La optimización estructural y constructiva que realiza del modelo de Jacobsen denota una enorme capacidad de para leer y encontrar los valores de la arquitectura contemporánea (Figura 8).

Se reproduce el esquema de aulas trabadas por su lado más corto, suprimiendo el patio y escalonando la edificación, para que las aulas se sitúen descabalgadas media planta entre sí. Esta habilidosa maniobra le permite compactar extraordinariamente la edificación, reduciendo la superficie de fachada y las circulaciones al minino.

Esta estrategia le permite alcanzar una arquitectura austera, económica y modulada, sin renunciar a la calidad espacial de las aulas, que son el espacio fundamental del proyecto docente. Todas iguales, perfectamente orientadas, iluminadas y ventiladas a través de un ventanal alto continuo, que permite ver el cielo sin más referencia.

Este ventanal alto concentra en un único elemento la capacidad del arquitecto de poner énfasis y apostar por aquello que considera irrenunciable del proyecto.

Mediante la reinterpretación para nuestra latitud de los huecos altos de la arquitectura de Jacobsen, la luz natural que se introduce en el aula es homogenea, evitando el deslumbramiento de los alumnos y garantizando ahorro energético en las horas de docencia.

Entender esta relación con la luz y con el exterior, es el argumento que construye el espacio del aula, y con él toda la escuela.

García de Paredes concibe un elemento prefabricado de hormigón que forma las ventanas y que soluciona la estructura, que finalmente vertebra y construye todo el edificio. 

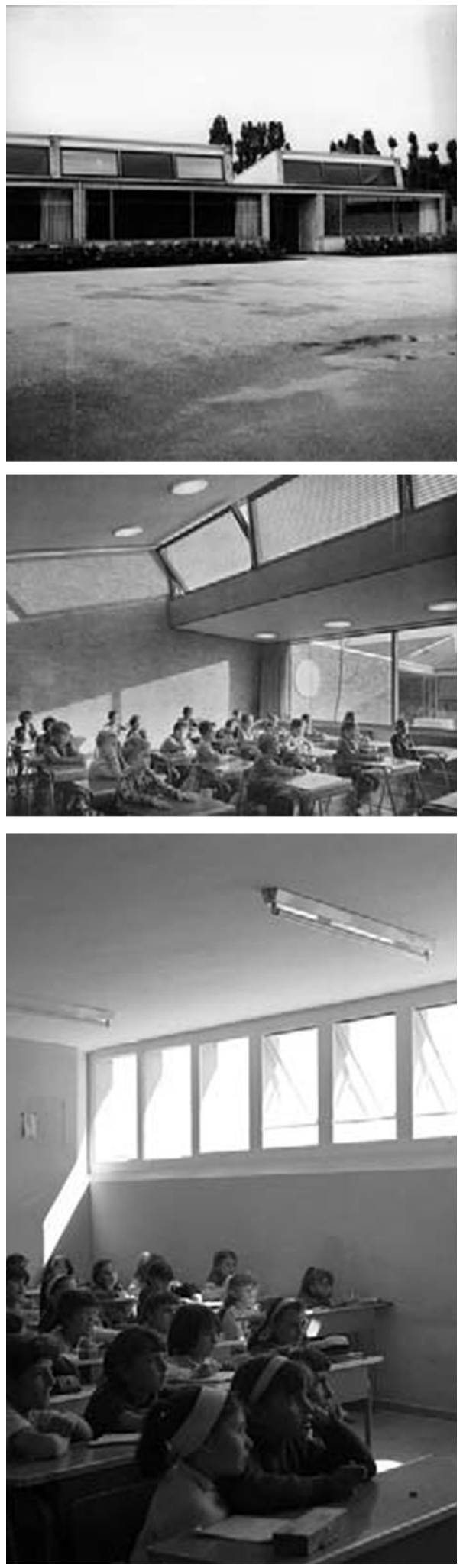

Figura 7. Escuela Munkegards, 1957 -fotografía JMGP-. Aula en la Escuela Munkegards de Jacobsen, 1957 -foto de la monografía de Johan Pedersen-. Aula de la Escuela de Zaidín, 1957 -fotografía JMGP- (4).

Por medio del sistema constructivo de compactación y escalonamiento, posibilita a su vez que en la planta baja se liberen espacios para los usos comunes y compartidos. Usos como laboratorios, aseos, y despachos de profesores, el salón de actos-capilla. Este último por estar en el corazón de la planta tiene planta y media de altura, y se ilumina a través
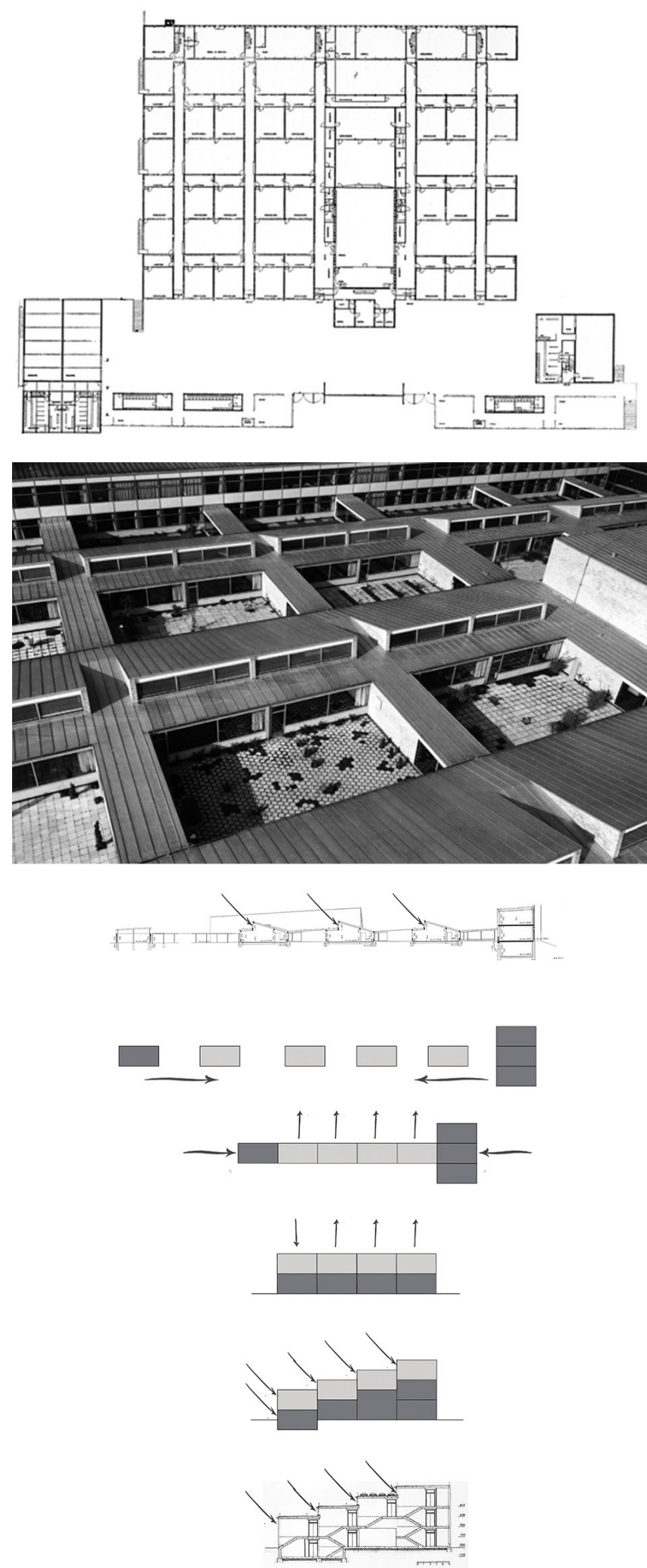

Figura 8. Escuela Munkegards, Arne Jacobsen 1951-1958 (10). Planta y esquema de evolución del modelo en sección (Esquema elaborado por el autor)..

de un lucernario que llega a cubierta a través de las aulas superiores.

«Se eligió como orientación deseable el cuadrante Naciente Mediodía, que evita el excesivo soleamiento en verano y que, por otra parte, era el que mejor se adaptaba a la orientación 
general del emplazamiento. Para lograr esta dirección solar óptima en la mayor parte de las aulas, se escalonaron éstas media planta, de manera que cada crujía recibiera iluminación y soleamiento por encima de la cubierta de la anterior» (8).

La galería de acceso a las aulas se reduce, minimizando de esta manera los costes de la edificación, este espacio deja de ser un pasillo, resultando un espacio de una enorme atractivo, que establece relaciones visuales entre los distintos niveles y generando un lugar de encuentro entre los escolares.

\section{LA CONSTRUCCION DE LA ESCUELA}

Las escuelas en Granada encierran un discurso constructivo íntimamente ligado al proyecto, que hace posible su ejecución en apenas un verano, y en una ciudad en la que los medios y técnicas con los que contaba eran escasos.

Se simplifican al máximo todas las instalaciones centralizando los servicios y las zonas de uso común.

El programa conduce lógicamente a un planteamiento de absoluta simetría en que los espacios de uso común ocupan una posición central. Igualmente se centralizan los aseos para conseguir una red de saneamiento y fontanería lo más corta y económica posible (8).

El sistema estructural mediante muros portantes de bloque cerámico, permite realizar el esquema escalonado de las distintas naves y forjar en el sentido más eficiente, para lo cual los ventanales se construyen en hormigón armado, con un solo elemento soluciona la entrada de luz y la sección que cubre el espacio docente.

El esquema arquitectónico adoptado y la rapidez con que había que levantar el centro se reflejan en el sistema constructivo como directa consecuencia: muros portantes de bloque cerámico, forjados de viguetas de hormigón pretensado y ventanales resistentes prefabricados. Todo el sistema se dispuso modulado en múltiplos de 30 y 70 centímetros, dimensiones de las piezas de pavimento y separación de viguetas con la que coincidía, para mejor reparto de cargas, la anchura de los ventanales de hormigón.

La ortogonalidad de los ejes de estructura garantiza el arriostramiento del conjunto, necesario en una zona altamente sísmica, mientras que la solución de ventanales resistentes asegura la continuidad lineal de los esfuerzos desde el apoyo de forjado hasta la zanja de cimentación, evitando vigas de gran luz en el sentido de la mayor dimensión del aula (8).

Resuelto el sistema estructural el espacio del aula está completamente definido, los acabados son austeros: terrazo, pintura blanca y una pizarra.

Las pocas particiones de tabiquería se realizan con medio pie de ladrillo.

En las tres escuelas el sistema estructural y de compartimentación es el mismo, pero la fachada y la cubierta varían para adaptarse a cada circunstancia.
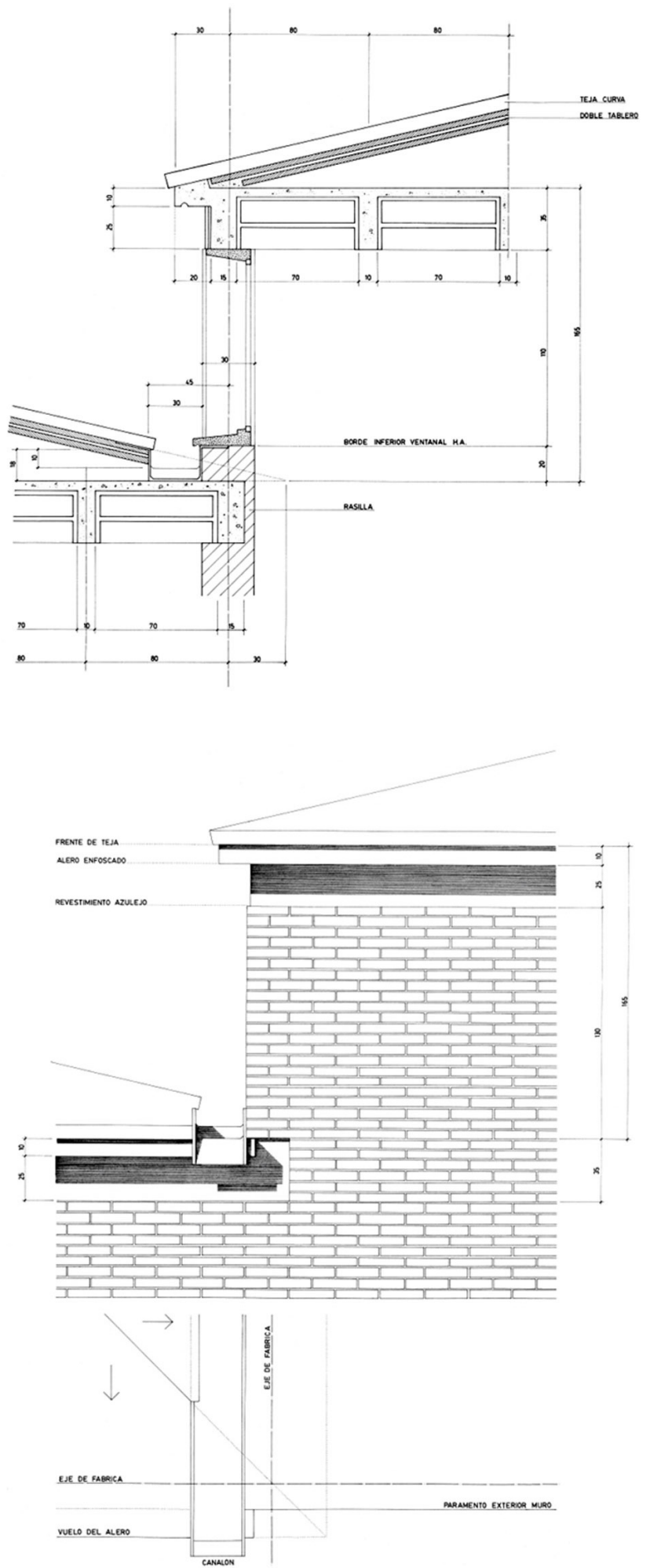

Figura 9. Centro de Enseñanza Cristo Rey en el Albaicín con cubiertas inclinadas. Detalles constructivos de canalón y ventana (11).

En el Zaid la Chana las cubiertas son planas y el muro portante de fachada se acaba mediante mortero y pintura blanca, mientras en el Albaicín las cubiertas se resuelven inclinadas sobre tabiques palomeros y teja árabe y el muro portante se proyecta en ladrillo visto (Figura 9).

Desde los primeros dibujos el esquema del edificio está planteado, evolucionando para ajustar los costes, optimizar la estructura y cumplir los plazos exigidos. 
García de Paredes parece resolver este proyecto como un ejercicio matemático, por el camino más corto, con la máxima elegancia. El arquitecto y la arquitectura al servicio de un fin mucho más importante, en el que su labor es hacer el proyecto lo más preciso posible, para que el resultado final añada nuevos valores no demandados por el encargo a un edificio, que en su humildad trabaja con altas expectativas.

Después de tanta premisa puede suponerse que no quedo lugar para filigranas: no había más que aceptar la forma, tal como era, y elegir el color de la pintura. Y fue blanco, porque no podía ser de otro color en la vega de Granada (8).

\section{CENTRO DE ENSEÑANZA MEDIA CRISTO REY. ALBAICIN}

La primera escuela construida en el Barrio del Zaidín se consideró todo un éxito, no solo porque cumplió con lo apretado de las fechas y de los costes, también por la claridad de sus espacios y la sencillez y austeridad de su propuesta. Esto hizo que la institución Juan XXIII y los usuarios lo hicieran suyo desde el primer momento.

Ese mismo año se construye un tercer colegio en Granada a partir del mismo esquema, pero en esta ocasión, la adaptación del prototipo a un entorno urbano complejo como es el del barrio del Albaicín, merece atención especial .

El encargo de este edifico lo realiza la comunidad religiosas hijas de Cristo Rey, para realizar una centro de enseñanza en un solar de su propiedad, para escolarizar 400 niñas.

El modelo ya ejecutado demuestra gran capacidad de adaptación y flexibilidad. García de Paredes proyecta y construye una variante del mismo esquema reinterpretando el lenguaje de las cubiertas inclinadas de teja árabe y la utilización el ladrillo para adaptarse a las condiciones del lugar.

El solar en que se emplaza el edificio es de forma muy irregular con una superficie de $4179,55 \mathrm{~m}^{2}$; linda al NW con la carretera de Jerez a Cartagena, al SW con el Callejón de las Cuatro Esquinas, al SE con la Calle de San Gregorio Alto, y al NE con el edificio con el edifico de las Religiosas Hijas de Cristo Rey. La orientación es aproximadamente NE-SW en el sentido de la mayor longitud del terreno. Se proyecta una Sección Filial femenina para 400 plazas (11).

El centro de Enseñanza Cristo Rey desarrolla por lo tanto la misma distribución de aulas escalonadas media planta. La solución se modifica para adaptarse a un programa diferente y a un solar acotado, irregular y con desnivel.

La escuela se encaja en una pequeña parcela, entre la carretera de Murcia y la calla San Gregorio Alto, y consiste en un volumen principal de aulas, orientado al sur-este, de manera que la iluminación de las mismas define la orientación y forma de todo el conjunto (Figuras 10 y 11). Este volumen principal está articulado con un eje central de escaleras-corredor, en el que se producen los diferentes accesos en los distintos niveles. En este caso por tratarse de un número menor de aulas, el acceso y el salón de actos se generan en un eje perpendicular y construyendo volúmenes adosados al volumen principal.
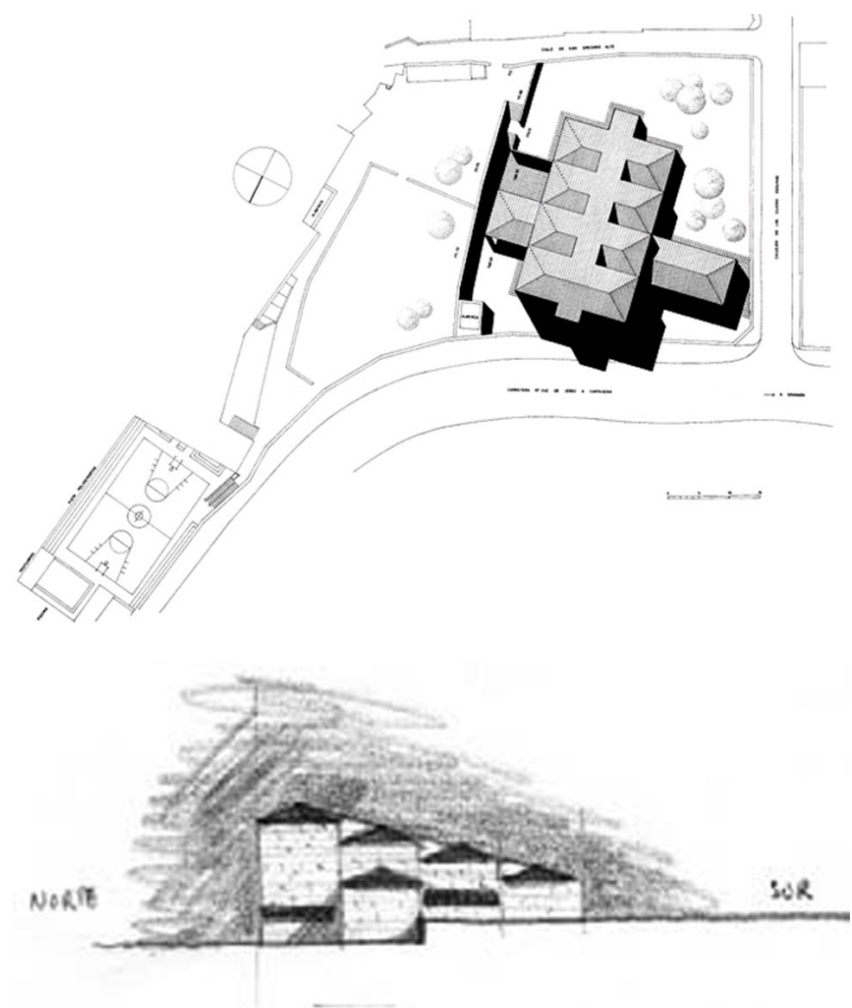

Figura 10. Centro de Enseñanza Cristo Rey, croquis (7) y emplazamiento (11), 1967.

El emplazamiento del edificio en pleno corazón del Albaicín, aunque no muy visible desde la Alhambra, obliga a considerar como factor determinante la necesidad de armonizar un edifico actual para la enseñanza con un conjunto arquitectónico de primer orden en el aspecto histórico, artístico y paisajístico. El programa de necesidades que hay que satisfacer conduce lógicamente a un planteamiento de simetría formal sobre dos ejes ortogonales, en el que los espacios comunes ocupen una posición central(11).

El esquema de aulas escalonadas se mantiene como principal articulador de los espacios, incorporando el lenguaje de las cubiertas inclinadas dentro del sistema, de manera que la galería de escaleras central queda cubierta por un único plano continuo e inclinado de teja árabe. Este plano único va enlazando y unificando en un único volumen las cubiertas de los distintos volúmenes escalonados, introduciendo luz por los hastiales a este espacio corredor central, dotándolo de una gran espacialidad (Figuras 12 y 13).

Se considera de importancia primordial la orientación de las diferentes unidades escolares: se ha elegido como óptima, la orientación al cuadrante Naciente-Mediodía, para evitar el excesivo calor en verano, y que, por otra parte, es la mejor se adapta a la orientación y planimetría del terreno. Para obtener esta orientación óptima en el máximo de los locales de enseñanza, se parte del principio de escalonar las aulas media planta de manera que cada una reciba iluminación y soleamiento por encima de la cubierta de la anterior. Este principio produce, además, una galería de distribución corta que va ascendiendo media planta en cada crujía, desde una en la fachada Sur hasta un total de tres en la fachada Norte. De esta manera se consiguen ocho aulas orientadas a SE, dos a E y W, y las aulas-laboratorio a estas mismas orientaciones (11). 

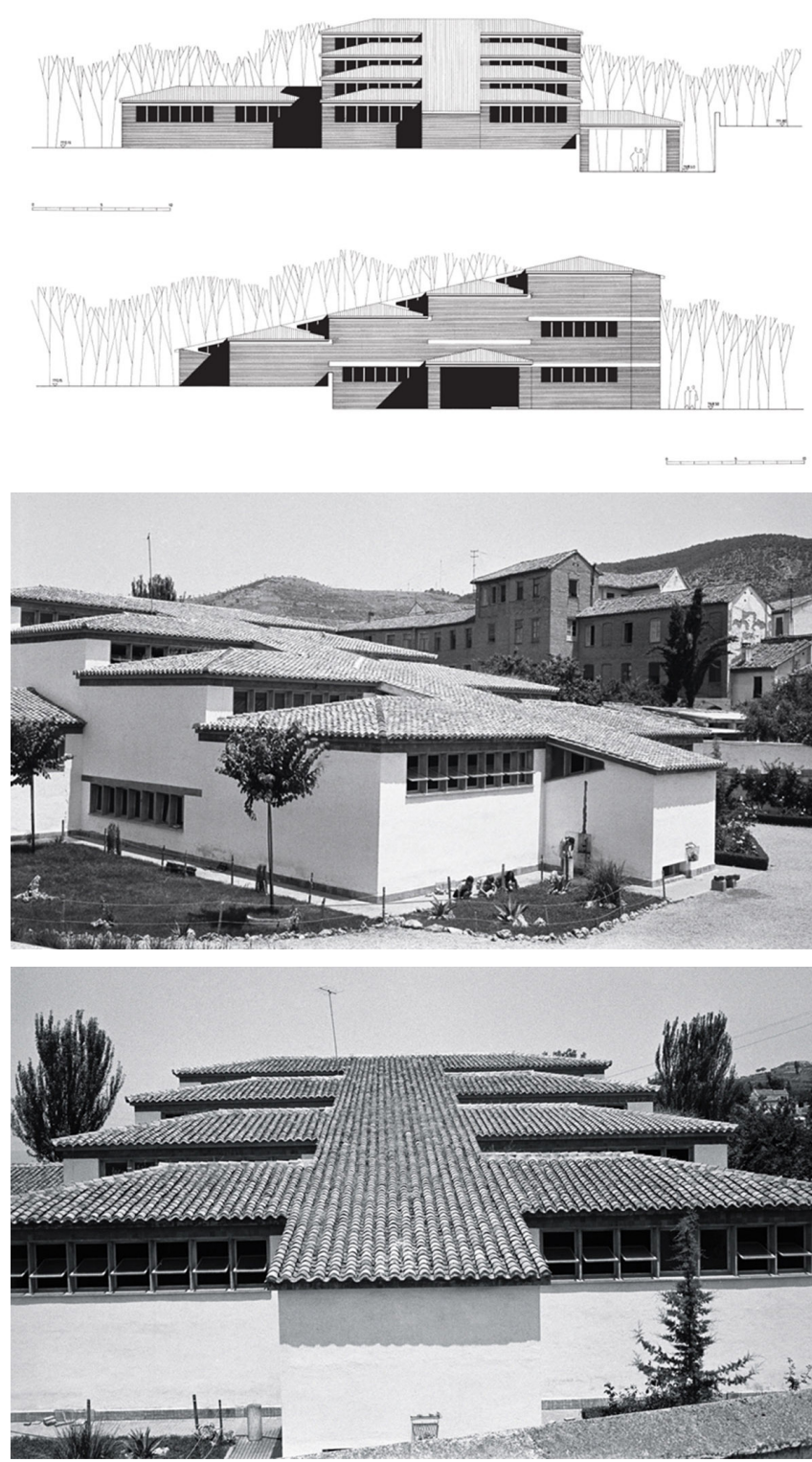

Figura 11. Centro de Enseñanza Cristo Rey en el Albaicín, alzados y fotografías de la época (11).

La renuncia a la cubierta plana, impuesta desde la ubicación del edificio, no resta ni un de modernidad al mismo; por el contrario es una muestra de la capacidad para introducir los lenguajes tradicionales dentro de la arquitectura de García de Paredes.

El resultado es absolutamente moderno, consiguiendo que el edificio quede inserto dentro de su entorno, de manera que el discurso no pierde el rumbo en cuestiones estilísticas.

En estas cubiertas vemos un adelanto de esta capacidad de trabajar con la cubierta de teja árabe, como un elemento más de su lenguaje, solución que veremos años después en el auditorio Manuel de Falla.

La disposición escalonada combinada con la topografía del terreno, produce en tercera crujía una altura de planta y media a nivel del acceso, adecuada para vestíbulo y sobre todo para gimnasio al permitir ventanales altos y colocación de espalderas bajo los mismos en el perímetro del local.
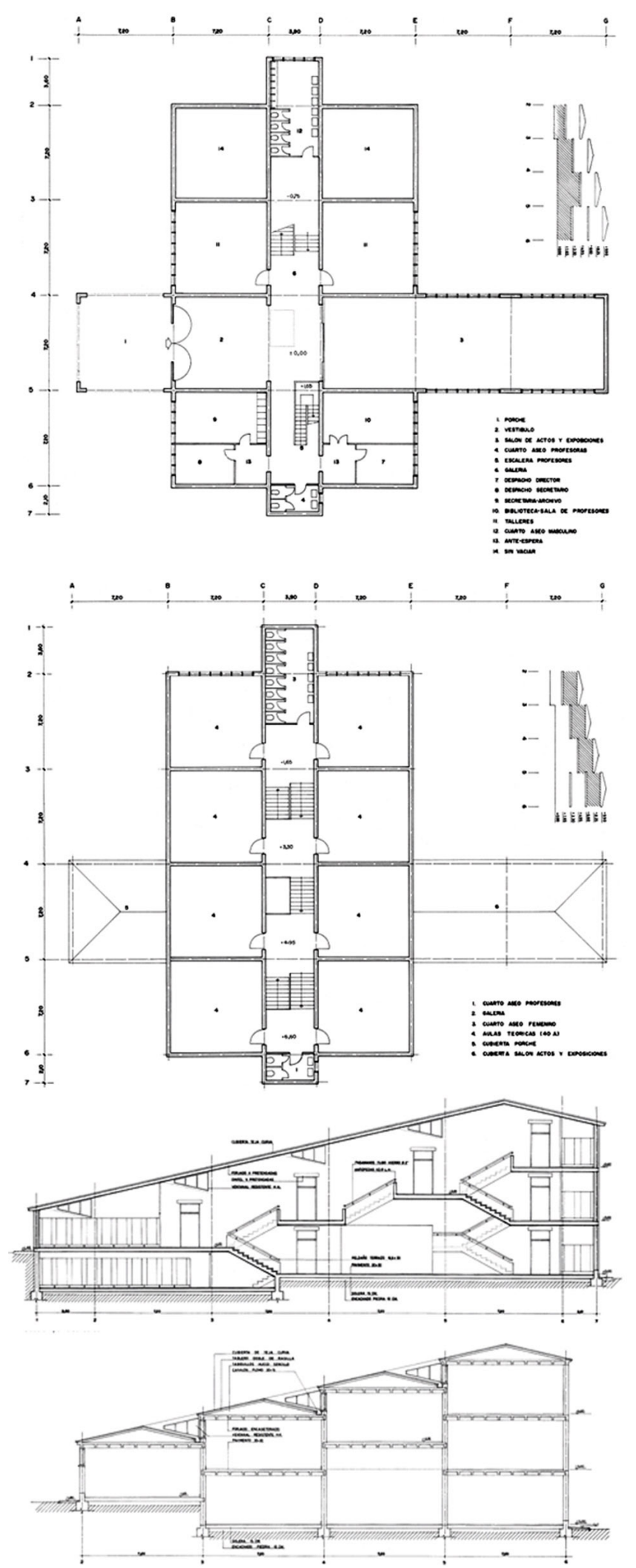

Figura 12. Centro de Enseñanza Cristo Rey en el Albaicín, con cubiertas inclinadas, plantas baja y primera y secciones longitudinales (11).

Se completa el proyecto con los locales de dirección y profesores situados en planta baja próximos al acceso principal y un porche cubierto para juegos en días de lluvia.

En el tratamiento externo del edificio se ha partido de la necesidad de conjugar la expresión propia de un edificio escolar 

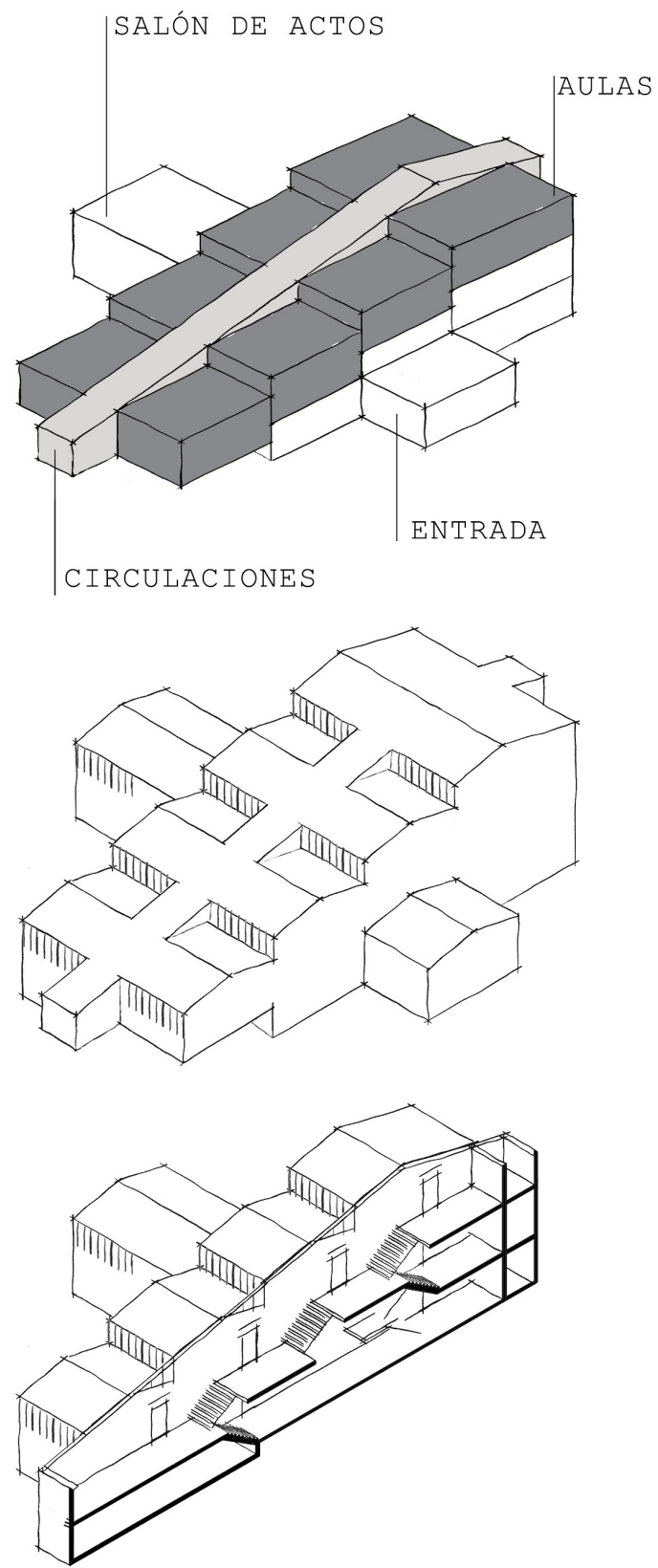

Figura 13. Centro de Enseñanza Cristo Rey esquema de uso y funcionamiento. (Realizado por el autor). proyectado en pleno siglo XX, con el ambiente exterior creado por una zona de la importancia plástica y paisajística del Albaicín. Para ello se ha realizado un estudio muy detenido de las características esenciales granadinas que determinan la expresividad de este conjunto urbano para manejarlas libremente en la composición del edificio proyectado (11).

\section{CONCLUSIONES}

José María García de Paredes, construyó entre 1964 y 1967 tres escuelas en Granada, una arquitectura comprometida, que sigue sorprendiendo cincuenta años después por la vigencia y rotundidad de los principios que las originaron.

Estos son los únicos edificios escolares que García de Paredes construyó en su carrera, en ellos sintetizo un discurso completo sobre los nuevos espacios docentes que se demandaban por la sociedad de la época. Y lo hizo en unas circunstancias especialmente complicadas en las que el proyecto surge como solución a un problema casi imposible de tiempos, economía y construcción.

Aunque nunca fueron pensadas para estar en servicio tantos años, y fueron construidas con materiales humildes, las escuelas que se construyeron en Granada entre 1964 y 1967, para una población escolar total de 2400 estudiantes, han cumplido de una manera ejemplar los cincuenta años, y lo hacen en pleno funcionamiento, sin haber sufrido graves modificaciones en las formas y principios que las generaron.

Desde su inauguración, han formado parte de la vida de los barrios en las que han desarrollado una función docente ejemplar. En estos años, los emplazamientos han variado radicalmente, de estar situadas dentro de la vega a estar rodeadas por la circunvalación, lo que no les ha restado que sigan percibiéndose como arquitecturas precisas, llenas de sabiduría y conocimiento, que han sido una pieza fundamental para mejorar la vida las personas de estos barrios.

José María García de Paredes es un arquitecto que se descubre de una manera personal y nueva en cada acercamiento que realizamos a sus obras o escritos. Un arquitecto capaz de construir un discurso nítido y deliberadamente silencioso, en el que el arquitecto siempre se mantiene en un segundo plano.

\section{REFERENCIAS}

(1) Flores López, C. (1961). Arquitectura Española Contemporánea. Madrid: Aguilar.

(2) Mosquera, E., Pérez, M. T. (2001). Cámara de Comercio e Indústria. Córdoba, 1950-54, p.52. Almería: Colegio Oficial de Arquitectos.

(3) Esteban Maluenda, A. (1999). La vivienda social española en la década de los 50: Un paseo por los poblados dirigidos de Madrid. Cuaderno de Notas, (7): 55-80.

(4) García de Paredes, A. (2015). La arquitectura de José M. García de Paredes. Ideario de una obra (Tesis Doctoral). Madrid: ETS Arquitectura - Universidad Politécnica de Madrid.

(5) VVAA. (2011). José María García de Paredes: Iglesia y convento de Santa María de Belén Stella Maris. Colección AACC vol. 10. Pamplona: T6 Ediciones, Escuela Técnica Superior de Arquitectura-Universidad de Navarra.

(6) Hernández Soriano, R. (2001). José María García de Paredes en Granada 1962-199o (pp. 32-34). Granada: Colegio Oficial de Arquitectos de Granada.

(7) García de Paredes, A. (2015). La arquitectura de José M. García de Paredes. Ideario de una obra (Tesis Doctoral), p.116. Madrid: ETS Arquitectura-Universidad Politécnica de Madrid.

(8) Hernández Soriano, R. (2001). José María García de Paredes en Granada 1962-199o (pp. 34-52). Granada: Colegio Oficial de Arquitectos de Granada. 
(9) García de Paredes, A. (2015). La arquitectura de José M. García de Paredes. Ideario de una obra (Tesis Doctoral), p.114. Madrid: ETS Arquitectura - Universidad Politécnica de Madrid.

(10) Solaguren, F. (coord.). (1999). Arne Jacobsen. Edificios públicos. Revista Internacional de Arquitectura 2G, (4): 44-45.

(11) García de Paredes, J. M. (2011). Centro de enseñanza media Cristo Rey, 1965 Albaicín, Granada. Revista Conarquitectu$r a$, (40): 60-74. 